\title{
Objective measurement of facial neuromuscular retraining therapy combined with botulin toxin injection for facial paralysis
}

\author{
Joo Hyun $\mathrm{Kim}^{1}$ and Jin $\mathrm{Kim}^{2}$ \\ ${ }^{1}$ Yonsei University College of Medicine, Yongin Severance Hospital \\ ${ }^{2}$ Inje University College of Medicine
}

July 5, 2020

\begin{abstract}
Objective: We hypothesized that neuromuscular retraining therapy combined with botulinum toxin injections (NMRT-B) could enhance the effectiveness of treatment for facial problems caused by facial paralysis. Also, we developed a new method to evaluate facial functions in a detailed, accurate, and objective manner. Methods: It has been recommended that patients with one-sided facial paralysis that does not resolve for more than 6 months undergo scheduled treatment with NMRT-B. The patients were scheduled to begin NMRT after 1-2 weeks of botulinum toxin A injection on the affected side and/or on the unaffected side. The second botulinum toxin A injection was conducted at 6 months after the first injection, and NMRT was continued for more than 6 months. And we used a computer-based numeric scoring system alone to confirm facial functions. Results: Most patients with chronic facial paralysis who underwent NMRT-B treatment exhibited better results after 1 year. NMRT-B provided satisfactory control of synkinesis as well as primary movement. Using our method for evaluation of facial asymmetry, the average primary facial function movement score was calculated as 57.3 (0.573), average secondary facial function movement score was $14.2(0.142)$, and average final facial function movement score was 43.1 (0.431) before treatment Conclusions: We demonstrated that NMRT-B was effective for patients with synkinesis and facial asymmetry and that the results differed depending on the degree of facial paralysis. We also established a method to evaluate facial paralysis in an objective manner that excludes subjective judgment.
\end{abstract}

\section{Hosted file}

main text (ver1.).doc available at https://authorea.com/users/339868/articles/466685objective-measurement-of-facial-neuromuscular-retraining-therapy-combined-with-botulintoxin-injection-for-facial-paralysis 


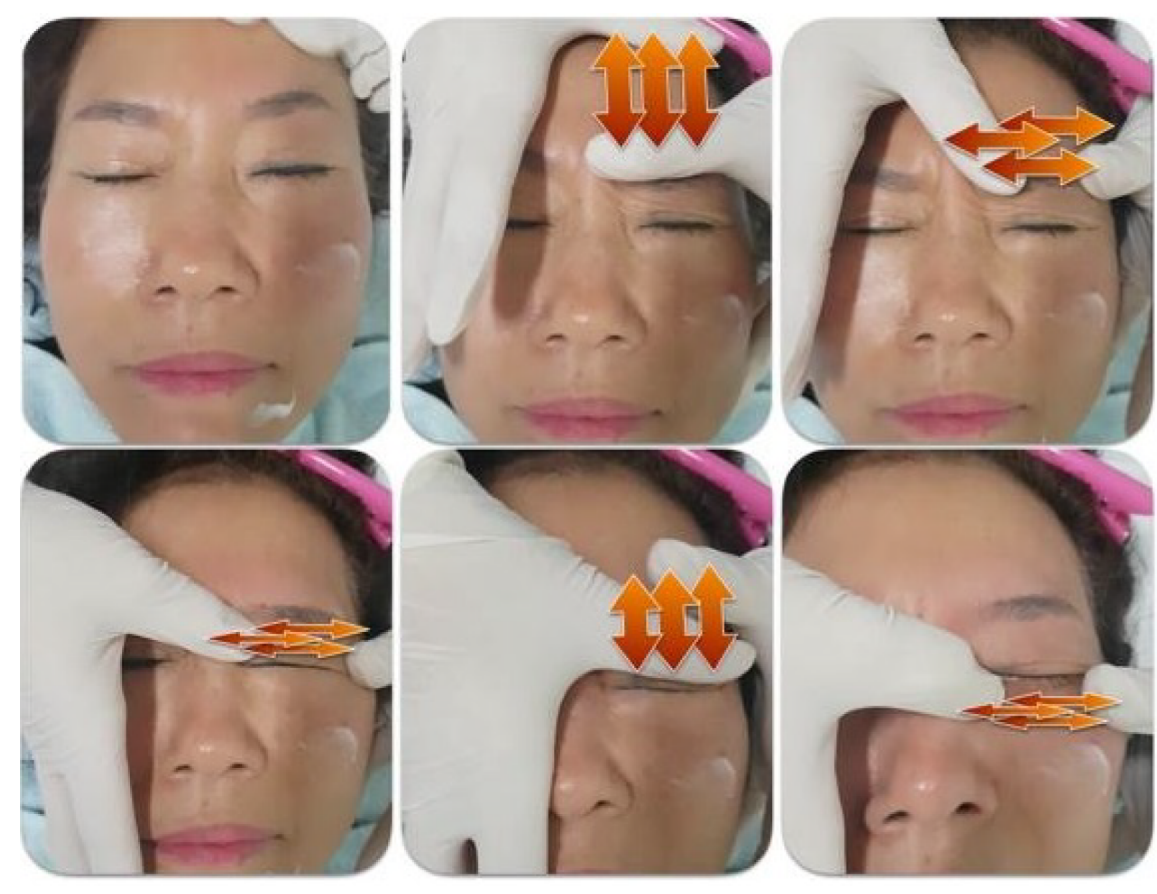

figures/fig2-A, B, C/fig2-A, B, C-eps-converted-to.pdf 
Analysis of facial asymmetry
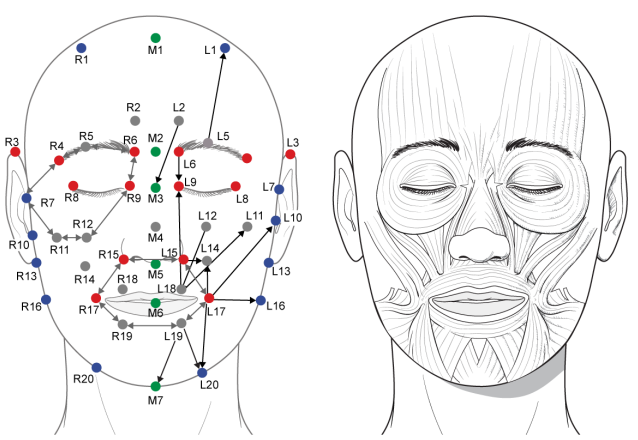

\begin{tabular}{|c|c|c|}
\hline MUSCLE & \multicolumn{2}{|c|}{ LOCATION (origin-insertion) } \\
\hline Frontalis & R1-R5 & L1-L5 \\
\hline Corrugator & M3-R2 & M3-L2 \\
\hline Depressor supereiliti & R9-R6 & L9-L6 \\
\hline Orbicularis oculi & $\begin{array}{c}\text { (R4-R7)+(R4-R5)+ } \\
\text { (R5-R6)+(R6-R9)+ } \\
\text { (R9-R12)+(R11-R12)+ } \\
\text { (R7-R11) }\end{array}$ & $\begin{array}{c}\text { (L7-L4)+(L4-L5)+ } \\
\text { (L5-L6)+(L6-L9)+ } \\
\text { (L9-L12)+(L11-L11)+ } \\
\text { (L11-L7) }\end{array}$ \\
\hline Levator labii superioris & R9-R18 & L9-L18 \\
\hline Nasals & R14-R15 & L14-L15 \\
\hline Zygomaticus minor & R11-R18 & L11-L18 \\
\hline Zygomaticus major & R10-R17 & L10-L17 \\
\hline Risorius & R16-R17 & L16-L17 \\
\hline Levator anguli oris & R14-R17 & L14-L17 \\
\hline Orbicularis oris & $\begin{array}{c}\text { (M5-R15)+(R15-R17)+ } \\
\text { (R17-R19)+(R19-M6) }\end{array}$ & $\begin{array}{c}\text { (M5-L15)+(L15-L17)+ } \\
\text { (L17-L19)++(L19-M6) }\end{array}$ \\
\hline Depressor anguli oris & R20-R17 & L20-L17 \\
\hline Depressor labii inferioris & R20-R19 & L20-L19 \\
\hline Mentalilis & M7-R19 & M7-L19 \\
\hline Exceptions & $\begin{array}{c}\text { Temporalis, Procerus, Depressor septi nasi, } \\
\text { Dilator naris anterior, Masseter, Buccinator, platysma }\end{array}$ \\
\hline
\end{tabular}

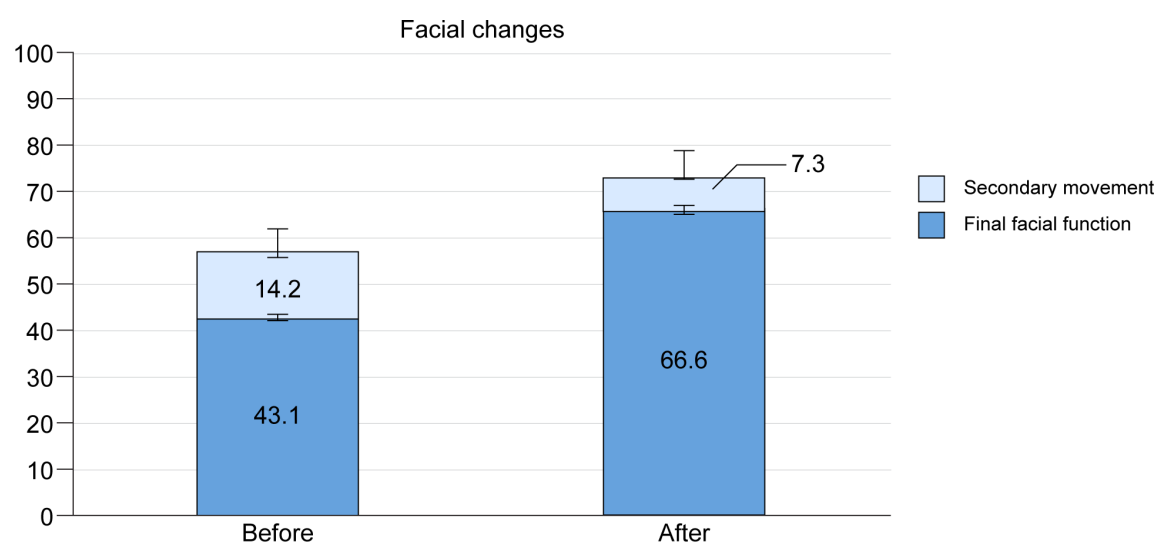


A

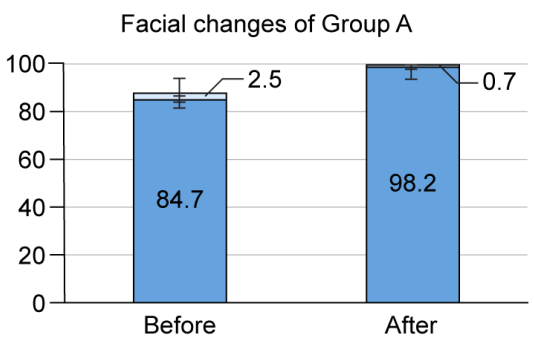

C

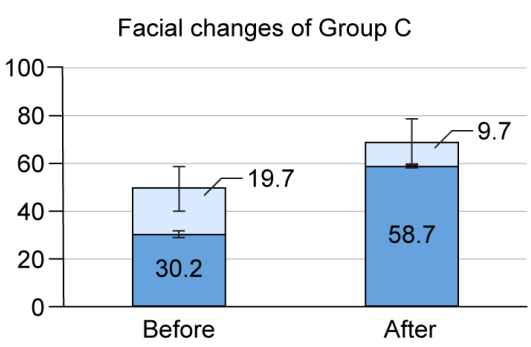

E

Facial changes of Group E

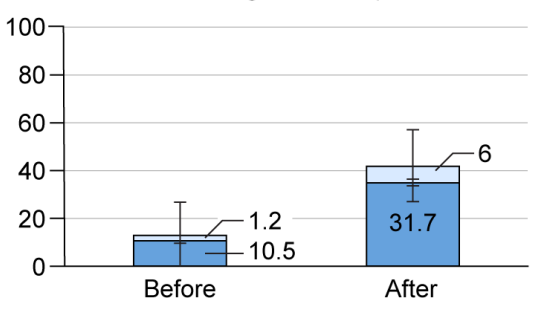

B

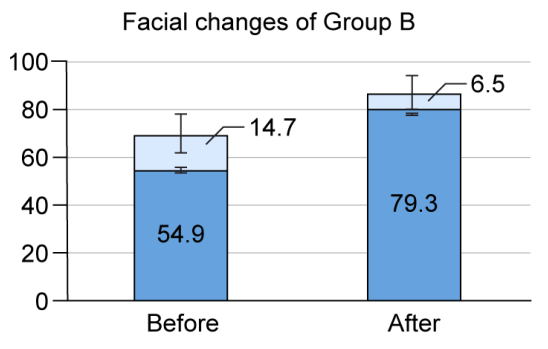

D

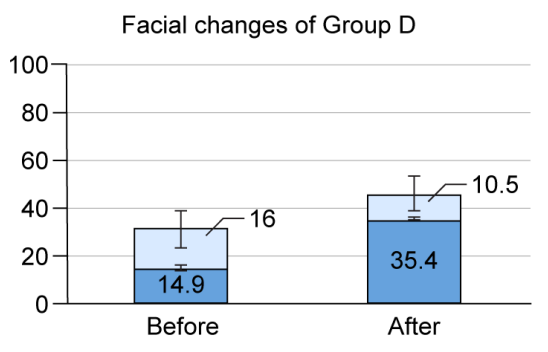

$\square$ Secondary movement

$\square$ Final facial function 
A

Percentage of seconday movement in facial function Before NMRT-B

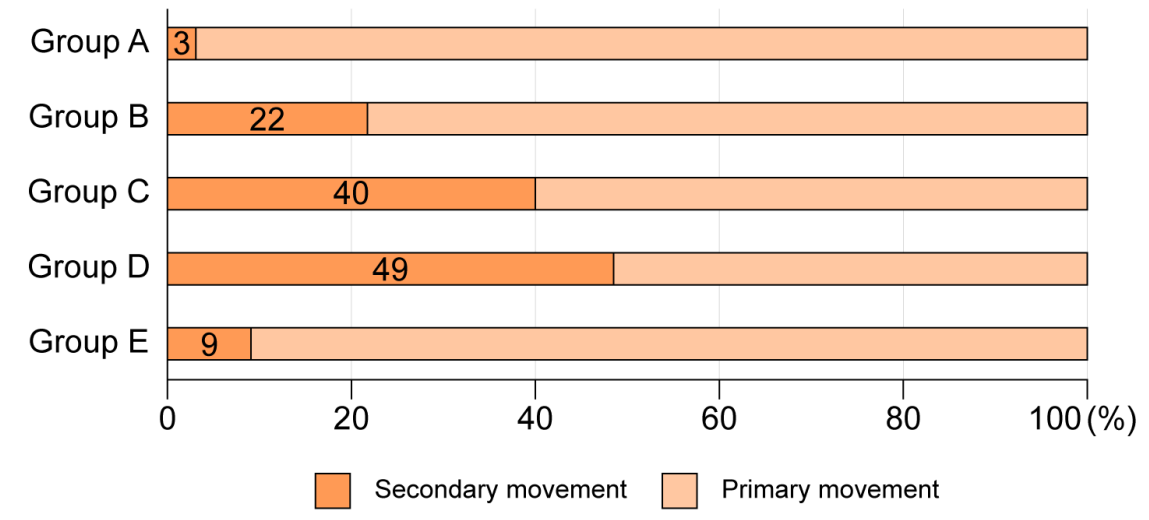

B

Percentage of seconday movement in facial function After NMRT-B

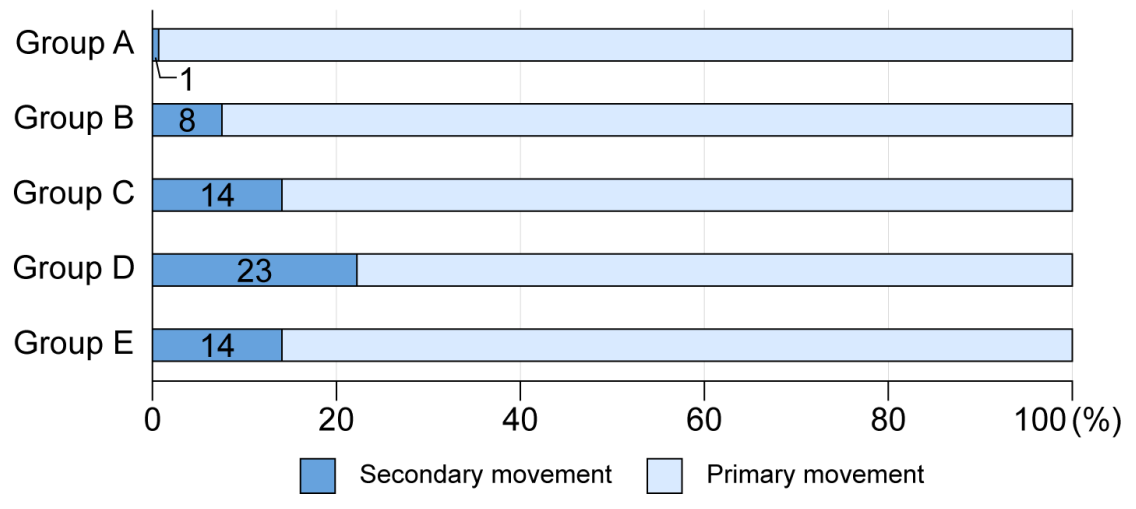

